Adventure and Ecotourism Safety in Queensland: Operator experiences and practice

Bentley, Tim A., Cater, C., Page, Stephen J.

Author's final refereed version, full text available from:

http://hdl. handle. net/1893/997

To be published in Tourism Management by Elsevier, 2009:

http://www.sciencedirect.com/science/journal/02615177 


\title{
Adventure and ecotourism safety in Queensland: Operator experiences and practice
}

\begin{abstract}
This study identifies the client injury experience and safety management practices of Queensland adventure and ecotourism operators, and to compare these findings with those from recent New Zealand surveys. The effectiveness of an on-line survey for collecting safety information from operators is evaluated in relation to the future development of an industry safety monitoring system. Some 60 adventure and ecotourism operators were surveyed, while in-depth interviews were conducted with four further Queensland operators. Survey findings indicated a relatively low-level of reported incidents, with slips, trips and falls the most common incident type. Risk factors identified by operators related most frequently to adverse and changeable weather conditions and client skills and behavior, and a notable proportion of operators reported that they did not apply important safety management practices. A model of injury control is presented to assist operators in their risk management practice.
\end{abstract}

Keywords: adventure tourism; ecotourism; Queensland; Australia; tourism safety; tourist injury; safety management; risk management 


\section{Introduction}

Adventure and ecotourism are fast growing sectors of the international tourism industry, and are major draw cards for some destinations for international visitors seeking new experiences in challenging and scenic environments. Adventure tourism has been defined as the 'deliberate seeking of risk and the uncertainty of outcome' (Ewert \& Jamieson, 1989: 8), and certainly appears to satisfy tourists' desire to engage in 'at risk' or risk-taking behaviours (Hall \& McArthur, 1994; Morgan \& Fluker, 2006). As we can assume that the majority of adventure tourism participants do not wish to become injured or face an actual risk of harm to themselves, it falls to adventure operators to simultaneously provide safe and exciting experiences (Cater, 2006; Morgan \& Fluker, 2006; Ryan, 2003). To achieve this, hazards and risks need to be effectively managed by commercial operators providing adventure experiences. Ecotourism has a less clearly defined link to risk, commonly identified as a variation of "a spectrum of naturebased activities that foster visitor appreciation and understanding of natural and cultural heritage and are managed to be ecologically, economically and socially sustainable" (Tourism Queensland, 2003). Although risk and safety management is not explicitly mentioned in this definition, the increased participatory involvement of clients, frequently in unfamiliar and unpredictable environments, means that concerns with risk management are equally pertinent in this sector. Indeed, numerous authors have identified the blurred dividing lines between these activities in practice, suggesting umbrella terms such as adventure travel (Page and Connell, 2006), ACE (Adventure-, Culture-, Ecotourism) (Fennell, 1999) or NEAT (Nature, Eco, Adventure, Tourism) (Buckley, 2000). 


\section{Adventure tourism research: Australasian perspectives}

Both adventure and ecotourism sectors are significant to the Australian, and especially the Queensland tourism product, with its high proportion of outdoor and nature-based tourism experiences. According to International and National Visitor Survey statistics from $2006,36 \%$ of all international visitors (1.8 million inbound tourists) and 16\% of all domestic tourists (12.0 million tourists) to Australia participated in adventure activities during their trip (Tourism Queensland 2007a). The most popular adventure activities for both groups were bushwalking, water sports, surfing, snorkeling, and scuba diving (Tourism Queensland 2007a). Furthermore, it is not just the traditional European and North American tourists that are interested in these activities, as studies have pointed to the importance of active nature-based tourism for emerging markets, with Yu \& Weiler (2001) identifying ‘visiting national parks and nature reserves’ as second only to 'going to the beach' in priority for Chinese tourist activities whilst in Australia. Further, research conducted by Pearce (2004) on Chinese visitors to the Great Barrier Reef, suggests that they are more active and have a higher interest in learning than has been the experience with previous Asian markets.

Much of the research in this area on the experiences of adventure tourism has been undertaken in New Zealand, where it has become an important niche sector of the tourism industry, with large numbers of overseas visitors and residents participating in adventure and eco activities each year, in contrast to other countries where they are largely recreational and domestic tourism activities (e.g. the UK). Partly this is a result of New Zealand using its natural environment as central to its overseas tourism marketing from 2000 , evidenced in the ' $100 \%$ pure' campaign. As Page and Connell demonstrated, other destinations such as Scotland have since sought to develop this niche, although according to their research they would appear to be at a more evolutionary stage 
of the product lifecycle (2006:73). In a summary risk analysis for adventure tourism safety based on a decade of research in the New Zealand adventure sector, Bentley \& Page (2008) identified activities presenting greatest risk of injury to participants, key risk factors for client injury, and current risk management practices to address these risks. Highest ranked commercial adventure activities in terms of client injuries included horse riding and white water rafting, although the study found unguided/non-commercial adventure activities, notably mountaineering, tramping and mountain biking, to be the major injury concern for the adventure recreationalists in New Zealand. Slips, trips and falls (STF) were by far the most common type of injury for adventure and ecotourism operations, while underfoot/STF hazards, changeable weather conditions and clients not following instructions were the risk factors most frequently associated with client injury risk by operators. From this data, and a review of the literature, the authors presented a conceptual information processing model for adventure tourism safety, which highlighted the role of both latent conditions and active failures in client injury causation.

In contrast, research on adventure and ecotourism safety in Australia has tended to focus on specific activities and environments, notably dive tourism (e.g. Coxon, 2006; Garrod and Gössling 2007; Wilks, 2007; Wilks \& Davis, 2000), beach safety (e.g. Morgan, 2006; Wilks, de Nardi, \& Wodarski, 2007), and human interaction with potentially hazardous natural environments (Ryan, 1998; Mühlhäusler \& Peace, 2001; Peace, 2001; Weaver, 2002). Indeed, these studies provide detailed commentary on key tourist safety issues of particular concern to the Queensland region. The overall extent and nature of the Queensland adventure and ecotourism safety problem has been investigated in a number of epidemiological studies of overseas visitor mortality and morbidity, based on a range of data sources. One such study involved an analysis of 1513 overseas visitor deaths across Australia during the 4-year period, 
1997-2000 (Wilks, Pendergast, \& Wood, 2002). Of the 307 recorded accidental deaths $(20 \%$ of all overseas visitor deaths), the main causes were motor-vehicle (51\%) and water-related (24\%). Water-related deaths were comprised of accidental drowning and submersion, the largest category, and water transport accidents. Just 25 cases ( $8 \%$ of accidents) involved accidental falls, this figure being markedly low in comparison to that found for New Zealand where falls during adventure activities are by far the major type of injury (Bentley et al., 2008), reflecting the differences in environments and activities encountered by visitors to the two countries. While older visitors were responsible for the large majority of fatalities overall, largest accident counts were observed for the 20-29 age group, in line with New Zealand research (Bentley, Meyer, Page, \& Chalmers (2001), which is representative of the known age range for adventure recreation participation.

A further study, concerned with overseas visitor injuries in Queensland hospitals for the period 1996-2000 (Wilks and Coory, 2002), involved the analysis of admissions data for 161

Queensland hospitals. As in the case of visitor fatalities (Wilks et al., 2002), and previous Queensland hospitalization studies (Nichol, Wilks and Wood, 1996), motor vehicle crashes were the most common type of injury, comprising $22 \%$ of overseas visitor injuries, while falls on the level $(16 \%)$ were the next most common injury cause, in line with New Zealand hospitalisation research (Bentley et al., 2001). The authors also identified diving accidents as a leading type of injury-related incident for international visitors to Queensland, with a further analysis of waterrelated injuries reported by Wilks and Coory (2000) showing that decompression illness associated with scuba diving comprised $55 \%$ of water-related hospital admissions to overseas visitors. 
A further perspective on the Queensland tourism injury problem was achieved through analysis of Workplace Health and Safety Queensland reports on incidents involving visitors and staff for the period, 1999-2004 (Parfitt, 2006). Highest numbers of visitor/tourist incidents were identified for water transport, accommodation, and recreational environments. From qualitative reporting of incidents, it was possible to determine that the greatest proportion of visitor/tourist incidents occurred in scuba diving or snorkeling situations, with diving the most common activity, being a factor in 131 out of the 594 (22\%) incidents analysed. Sudden death, decompression illness, water ingestion and fall injuries were the most common outcomes of these incidents.

These Australian and Queensland epidemiological studies provide strong supporting evidence for the key role of diving and water-related activities in Queensland visitor morbidity and mortality. Analyses of Queensland Emergency Department injury presentations for the domestic population, however, indicate that adventure and eco-tourism activities contribute only in a very minor way to the overall domestic injury count in Queensland. For example, fishing and swimming were the most common activities for water-related injuries for the period 1998-2003, with drowning comprising just 1\% of cases (Scott, Hockey, Spinks, \& Pitt, 2004). Further, across Australia, non-adventure-related causes of 'high threat to life' unintentional injury dominate hospitalization data, with largest external causes of community injury cases for the 2004-05 period being falls $(32,076)$ followed by transportation $(13,566)$, while drowning cases totaled just 414 (Bradley and Harrison, 2008). Indeed, adventure sports injuries comprise just $0.5 \%$ of all injuries involving sporting activity for the same period, although other categories such as 'water sports' $(6.3 \%)$, equestrian activities $(4.6 \%)$ and aero sports $(0.4 \%)$ will contain cases that are commercial adventure tourism-related. These findings for the general population 
add support to the common finding that, unlike residents, overseas visitors are particularly susceptible to risks associated with outdoor, wilderness and marine-based activity, and are most likely to experience safety problems and injury in unfamiliar environments and when participating in unfamiliar activities (Bentley et al., 2001; Nichol et al., 1996; Page and Meyer, 1997; Wilks and Coory, 2002; Wilks and Watson, 1998).

The studies reported above also highlight the problem of accessing complete and accurate information on overseas visitor and inter-state tourist injury (Parfitt, 2006), and the fact that improvements in reporting and monitoring in this area should be a priority for government and industry. It is also noteworthy that while these studies provide a useful overview of key risk areas for prioritisation of preventive activities, notably improved risk management and visitor education and awareness, they say little about possible causation and risk factors for adventure and ecotourism incidents, nor help us understand the quality of current risk management activity across the wider tourism operator sector (Morgan and Fluker, 2006).

Commercial Queensland adventure and ecotourism operators are subject to mandatory legal requirements, notably the Queensland Workplace Health and Safety Act 1995, but also specific government licensing requirements and Codes of Practice (Morgan and Fluker, 2006), having a Duty of Care to employees and clients as employers and persons controlling a place of work or work area. Notably, operators must identify, assess and control hazards in order to manage exposure to risk of employees and clients. Further, Australian commercial operations are subject to damage claims from injury incidents and may have to pay compensation to those parties suffering loss or injury, particularly where their Duty of Care to clients is breached. This is in contrast to the situation in New Zealand, where Accident Compensation legislation (Accident Rehabilitation and Compensation Act 1992), administered by the Accident Compensation 
Commission (ACC), is a no-fault scheme providing cover for New Zealanders and overseas visitors, and protection to operators from damage claims from injured clients.

Many operations in Australia, like New Zealand, are run by one or two person owner-operators, who are responsible for all aspects of business management, including the management of safety, along with the day-to-day running of operations. Previous studies have indicated that an operational rather than strategic focus, time and cost constraints, and a lack of safety/risk management knowledge are barriers to safety activity for many smaller businesses operating in this sector (Bentley, Page, \& Walker, 2004). The level of safety management may also reflect the nature of the activity. For adventure operations where a high-level of risk is intrinsic to the activity, such as bungy jumping or sky diving, risk management will be an integral part of the operation. However, relatively low-hazard activities such as horse riding or wilderness hiking may not appear to require the same level of risk management attention from the operator, meaning insufficient controls may be adopted to prevent incidents and injuries to clients. In other words, a one size fits all approach does not seem appropriate for a diverse industry sector.

The present study builds upon the 1999, 2003 and 2006 New Zealand studies on adventure tourism safety in New Zealand, and seeks to explore the unique situation for Queensland operators. The study aims to explore the extent and nature of client injury in the Queensland adventure and ecotourism sectors, major incident types, and risk factors for client injury. In addition, the study aims to examine the safety and risk management practices of Queensland operators. A dual aim is to examine the potential usefulness of an on-line survey of operators in achieving client injury and safety management monitoring in the Queensland sector. A longerterm objective, towards which this study aims to contribute, is to move towards the eventual establishment of an industry-owned, Queensland adventure and ecotourism injury surveillance 
system, that will allow monitoring of patterns and trends in commercial adventure and eco tourist client injuries, and priorities for prevention to be established. A final aim of the present study is to compare Queensland adventure and ecotourism injury and safety management data with those of recent New Zealand surveys, and to contribute to a growing database of international adventure tourism data, with other recent studies using a similar survey undertaken in Scandinavia, Scotland, and New Zealand. It is hoped that such studies are beginning to address the lacunae identified by Page in the 1990s, where information on safety management was 'fragmented, scattered across a range of sources and not available in a manner which permits a systematic assessment of the issue' (1997:34).

\section{Methods}

\subsection{Operator On-line Survey}

The study surveyed businesses registered with Tourism Queensland's website, which included 182 adventure operators and 200 ecotourism operators. Operators were asked to respond to a Tourism Queensland newsletter invitation to participate in the on-line survey, although it is not possible to know exactly how many operators were aware of the survey and read the invitation to participate. The online website was open to respondents during the period, March 2008-May 2008. The survey invited operators working in adventure tourism, outdoor adventure recreation, ecotourism or adventure education to participate. An introductory statement to the on-line platform outlined the purpose of the study - which was to better understand the safety-related experiences and activities of Queensland adventure and ecotourism operators - and noted that a summary of findings would be presented in future Tourism Queensland publications. 
Respondents were also informed that their participation was confidential, and no responses would be attributed to any single individual or business.

The on-line questionnaire comprised three distinct sections: the business (ownership status, staffing issues and size of business, time in business, location of business, nature of activities provided, number of clients); safety management activities/practices (reporting and investigation of client injuries and incidents, safety and risk management systems in place); and a final section on client injuries and perceived causal factors (including number of minor, serious, fatal injuries recorded in the accident register (a legal document for the recording of injury incidents) for the preceding 12-month period: January-December, 2007). For the purpose of this questionnaire, serious harm injuries were defined as 'injuries requiring hospitalization, fractures, serious lacerations or burns, near drowning'; and minor injuries as 'cuts and bruises, etc. not requiring hospitalization'. This section also asked respondents to identify the most frequent type of incident (e.g. slips, trips and falls; striking against and object; drowning or near drowning), and risk factor perceptions for these incidents (selecting risk factors from lists under the headings: client-related factors, task and equipment-related factors, physical environment factors, and management/organization factors).

Each of these questionnaire items were derived largely from the New Zealand survey instruments, although some minor adjustment was made for the Queensland situation, and some new items included around aspects of safety management practice. This was done in order to allow for some comparison between New Zealand and Queensland samples in the present analysis, and to contribute to moves towards monitoring of the adventure sector internationally, as described above. 
Descriptive analysis of quantitative data was undertaken using SPSS for Windows (version 13) and consisted of frequency distributions and cross tabulations of categorical data, and calculations of means and other measures of central tendency for different grouping variables. Comparison was also made, where possible, with data from the most recent survey of New Zealand operators (Bentley, Page, \& Edwards, 2008).

\subsection{Method: Semi-Structured Face-to-Face Interviews}

The highly contextual nature of safety and risk management in adventure and ecotourism activities recognized by Cater (2006), requires a mixed methods approach in order to calibrate the empirical findings. Thus, a further four adventure tourism operators from the Gold Coast and Brisbane regions were surveyed through semi-structured, face-to-face interviews. Nonrespondents to the on-line survey were selected for the interviews to increase the overall operator participation in the survey $(\mathrm{n}=64)$, to survey some of the perceptions of non-survey respondents, and to gather in-depth knowledge of the safety management activities and practices of operators. The four respondents represented the bungy jumping, bridge climbing, whale watching, sailing, rock climbing, flatwater kayaking, and rollerblading sectors (two operators offered multiple activities). Each business had been in operation for a number of years and respondents each had at least two-years experience in the business. The respondent was the business owner or partner in two cases, and manager in two cases.

Interviews took place at the site of the adventure business, and took between 30 minutes to onehour to complete. Each interview was tape recorded at the permission of the respondent, and respondents were informed of their participants' rights according to the human ethics 
requirements of Griffith University, notably the confidential nature of their participation and their right to refuse to answer any question or withdraw from the survey at any point.

Interviews followed a semi-structured format, allowing for detailed follow-up on any issue. The interview schedule contained open questions on four themes which mirrored those used in the questionnaire survey: nature of business and activities provided commercially; client safety experience and perceptions of risk; safety management and risk management practices (general), and those activities specific to the activity provided. Analysis of interview data involved categorization of responses into a number of specific themes (thematic content analysis), in relation to the specific style of safety management employed by each operation.

\section{Results: Operator Survey}

\subsection{Survey Sample Characteristics}

A total of 66 Queensland operators responded to the online survey, although the analysis included data from 60 respondents due to missing data for six of the completed surveys. An accurate response rate could not be determined as it is not possible to know how many operators received notification about the survey (i.e. read the Tourism Queensland newsletter item or received notification through some other medium). However, taking the database categorization, this represents a minimum return of approximately $16 \%$. Although this figure is not high, the newsletter approach combined with online delivery was unlikely to achieve a much higher rate, and concurs with recent literature on internet based surveys. In cases where participants are directly targeted, and 'undeliverables' are removed from the sample, response rates for electronically conducted surveys often return relatively low response rates (e.g. 20.4\% ; Deutskens et al, 2004; 24\%; Trouteaud, 2004; and 25\% ; Kittleson,1997). It is also noted that 
the study was exploratory, and sought to determine the suitability of this method of gathering data from operators for on-going industry injury surveillance.

The study was very successful in terms of the geographical coverage of the survey, with the majority of responses from South East Queensland (Brisbane/Gold Coast/Sunshine Coast) (48\%), Tropical North Queensland (TNQ) (23\%), the Fraser Coast (12\%), and Townsville (5\%). Although these might seem to overweight TNQ and Fraser relative to the significance of tourism more broadly (13\% and 4\% of Gross State Product respectively (Tourism Queensland, 2007b)), the higher concentration of adventure and ecotourism operations in these areas means these responses are fairly representative.

The dominant pattern of ownership was either sole ownership (48\%) or jointly owned (42\%), with this distribution being very similar to that found in all New Zealand surveys of adventure tourism operations (Bentley et al., 2008). Most respondents described themselves as the owner of the business $(60 \%)$, manager $(12 \%)$ or employee $(21 \%)$. In terms of the age of businesses surveyed, adventure and ecotourism businesses had been in operation for a median duration of 11.5 years, with approximately $20 \%$ in business for 5 years or less and $45 \%$ for 10 years or less. As found in the New Zealand studies, most businesses surveyed were very small concerns, with the majority of businesses having 10 or less staff (80\%), while $67 \%$ had five or less staff, and $42 \%$ had just one or two staff members. However, the nature of employment contracting for most Queensland adventure and ecotourism employees appears to be different to those for New Zealand operators, as the majority of Queensland employees are not employed on a seasonal basis, with some $70 \%$ of businesses reporting that none of their employees were seasonal. Adventure and ecotourism operators responding to the survey reported a total of 774 employees, with a median of 3.5 employees per business. Employee turnover was not notably high for most 
operators, with $32 \%$ of respondents reporting zero turnover, and $53 \%$ of operators reporting a $5 \%$ or lower turnover rate. However, Queensland faces less of an issue with seasonality, with a far more favorable climate and stable pattern of tourists year-round than either Scotland or New Zealand (Tourism Queensland, 2007b; Statistics New Zealand, 2007; Visit Scotland, 2007).

Queensland tourism operators appear to be well represented by industry associations, with $79 \%$ of respondents identifying as having membership of one or more industry associations, and many belonging to several. Most frequently mentioned among these were Dive Queensland, the various regional tourism associations, and Queensland Outdoor Operators Federation.

\subsection{Activity and Client Distributions}

Respondents predominantly described their businesses as adventure tourism operations (50\%), ecotourism (35\%), or adventure education (10\%), using the definitions accepted by Tourism Queensland. A wide range of activity sectors were included in the sample of operators, and were most commonly water-based (38\%) or land-based (25\%). A further $12 \%$ were aviation-based, while $25 \%$ did not specify an activity or offered multiple activities. The 21 activity sectors included in the survey represent a wide range of adventure and ecotourism experiences, the large majority of which were active pursuits involving a moderate (e.g. bush walking) to high (e.g. scuba diving and kayaking) level of participation and some element of risk, although a number of relatively passive activities were also included in the sample (e.g. whale watching and scenic flights). The most common activity included in the analysis was scuba diving ( $\mathrm{n}=6$ operations; $10 \%$ ), followed by outdoor education, bushing walking and kayaking (all $n=3 ; 5 \%$ ).

Activities had a medium duration of five hours; a similar finding to that for New Zealand activities, which took an average six hours to participate in (Bentley et al, 2008). Some $12 \%$ of 
Queensland activities took one hour or less to complete, including travel to and from the activity site, and 53\% less than five hours, with a range of 15 minutes to several days participation. Operators reported a total of 344,312 participants during the 12-month period, January to December 2007, with client numbers ranging from 30 to 7000, and a medium of 2000 participants per operation. As found for New Zealand adventure tourism, operators reported male and female participants to be of fairly equal proportion (52\% male), although the proportion of female (horse riding, 85\%) and male (sailing, $80 \%$ and waterskiing, $70 \%$ ) participants were notably higher for certain activities. Respondents estimated that children younger than 16 years comprised $23 \%$ of participants, a notably larger amount than found for New Zealand operators (11\%). Some $41 \%$ of participants were estimated to be from Queensland, 26\% from interstate, and 34\% from overseas, with these figures differing significantly from those found for New Zealand operators, who reported a much higher proportion of overseas visitors $(62 \%)$.

\subsection{Perceived Risk Factors for Client Injuries}

Operators were asked to identify from a list of possible risk factors those factors that they believed to be the primary causes for accidents and incidents involving clients of their main activity. The risk factor list was generated largely from the findings of the New Zealand surveys, but included additional factors that were thought to be relevant to the Queensland context. Table 1 provides a summary of the most frequently identified risk factors, organized under the following subsystem headings: client-related causes, task and equipment-related causes, environment-related causes, and organizational/management-related causes. The table also shows the proportion of respondents identifying each factor in the most recent New Zealand survey of adventure tourism operators (Bentley et al., 2008). Despite the fact that these 
percentages are not directly comparable, we do see similar patterns in the most frequently identified factors.

\section{Table 1 about here}

From Table 1 it can be seen that two related factors: adverse or changeable weather conditions and unusual or unpredicted activity events, were of most concern as risk factors, although several other factors were also perceived as primary risk factors by respondents, notably those concerned with clients, including unfamiliarity with the environment and not following instructions. These factors were also identified as key causes of client injuries in New Zealand research, although to a much lesser extent, suggesting a possible point of difference between the two populations in terms of the nature of hazards related to client experience and behavior. Respondents for the Queensland study also identified sun exposure as an important risk factor, reflecting the hash outdoor conditions and problems of low client awareness of the problem of sun exposure (Peattie, Clarke, \& Peattie, 2005). As with the New Zealand studies, a number of respondents recognized the role of weaknesses in the organization of work and management of client safety as latent risk factors for client injury, although these factors were identified much less often than client and environmental factors.

\subsection{Injury Event Types (Mechanism of Injury)}

Respondents were asked to select the major types of injury events involving their clients that most commonly occur in actual incidents. The main injury event types (mechanism of injury) experienced by clients are shown in Table 2, along with corresponding findings from the most recent New Zealand survey of adventure operators. 


\section{Table 2 about here}

In line with previous New Zealand surveys and analyses of archival injury and compensation data (Bentley et al., 2001; Bentley et al., 2007), the most commonly reported events were 'underfoot incidents', including slips, trips and falls (STF) and stepping on/twisting ankle. These findings also reflect the high prevalence of STF among leisure injury data internationally, and the nature of adventure and ecotourism activities, many of which involve walking or climbing over a wide variety of terrains. Other common injury types included animal injuries - often involving animals not connected with the activity (e.g. insects), unlike the New Zealand findings where animal-related injuries were commonly from animals involved in the activities, notably horses (note, however, the impacts of midges on outdoor activity in Scotland highlighted by Blackwell and Page, 2003).. Surprisingly, exposure to heat or cold was identified by just two respondents, while this problem was more commonly noted by New Zealand respondents.

\subsection{Client Injury Experience}

Respondents were asked to record the number of minor, serious harm, and fatal injuries from their accident book or injury records for the 12-month period, January-December 2007. From the 47 respondents that answered this question (13 declined or put nonsense responses), a total of 253 injuries were reported, of which just 29 were serious harm (requiring hospitalization, fractures, serious lacerations or burns, near drowning) and two fatal. Minor injuries were recorded at an average of just under five injuries per operator, with some $26 \%$ of operators reporting no injuries to clients. Reporting rates were similar to those found for the most recent New Zealand survey, with a similar number of injuries reported per operator and an almost identical ratio of minor to serious harm injuries (Queensland: 222/29=7.7; NZ: 405/54=7.5), 
suggesting a reasonably healthy injury reporting culture among Queensland operators (i.e. operators report minor injuries at a rate of over seven for every one serious harm injury).

The study also considered the relationship between a range of business-related variables and reported client injuries. Of these, the only notable difference was found for injuries reported by size of business (ns), with larger businesses reporting the greatest proportion of injuries. Indeed, businesses with $>20$ staff reported $76 \%$ of injuries but represented just $49 \%$ of the sample. This finding is in line with those of New Zealand studies, and is most likely a reflection of the number of participants exposed to injury risk.

Finally, the analysis considered the reporting of injuries by region. Highest levels of reporting were found for Tropical North Queensland (ns) who reported 35\% of all injuries, but represented just $23 \%$ of the sample. Lowest proportions of client injuries were reported for the Sunshine Coast, Fraser Coast, and Townsville regions, although no differences were significant.

\subsection{Safety Management Activities}

Respondents provided information about a range of safety management activities used to control the risk of injury to clients. A formal risk management plan was in place in $80 \%$ of operations, compared to $92 \%$ for the New Zealand sample, while a formal emergency management plan was in place in $82 \%$ of cases. On the issue of reporting and investigating client injuries, approximately half of the sample (48\%) claimed to investigate all accidents, incidents, and nearmiss events involving clients, while a further $29 \%$ investigated all accidents resulting in actual injury. Unfortunately, some $23 \%$ of operators either investigated only serious harm injuries (requiring hospitalization, fractures, serious lacerations or burns, near drowning) or investigated no incidents at all. These figures do not compare well with those for the most recent New 
Zealand survey, where $76 \%$ of respondents reported investigating all accidents, incidents and near-miss events, while just $11 \%$ only investigated serious harm injuries or undertook no investigations.

Table 3 shows the proportion of respondents who reported undertaking a range of critical safety management activities. It is noted that at least three in four of the operators surveyed used risk assessment and regular maintenance checks of plant, vehicles and equipment, and safety training of staff/guides, while two operators reported using none of the activities.

\section{Table 3 about here}

Respondents were asked whether they would have all necessary safeguards and safety management systems in place if they were audited by a Workplace Health and Safety officer today. The majority of respondents $(80 \%)$ indicated that they believed they would, although $20 \%$ thought not $(7 \%)$ or were unsure $(13 \%)$. In response to the follow-up question, "is there any area of safety management that you believe this business should seek to implement or improve in the short to medium term", just over one-half of respondents $(52 \%)$ identified one or more activities, with safety audits or reviews being the most frequently mentioned activity (16\%), followed by hazard management, staff or guide training, and formal emergency procedures (all 11\%). Respondents were most confident about technical aspects of safety in their operation, notably maintenance checks on plant, vehicles, equipment, where just one operator felt improvement was required.

A final question on safety management asked operators whether they had access to the relevant information and/or assistance necessary to implement or improve the safety management 
systems in the business. Some $80 \%$ of respondents believed that such information and/or assistance was available, although $20 \%$ felt not or were unsure.

\section{Results: Semi-Structured Interviews}

\subsection{Approaches to Safety Management: Controlling Human and Technological Risks}

Approaches to the control of client risk were examined through questioning of operators on their safety and risk management practices. Respondents each described general risk management practices, including incident reporting and investigation, and the identification and assessment of risks and hazards associated with their activities, with responses generally consistent between the four operators, and in line with those for the wider survey population. However, it was apparent was that each operator employed quite different control measures as part of their risk management practice. Indeed, the analysis of respondents' comments revealed various combinations of technological and behavioral control used to achieve client safety. For example, the bungy operator described high-levels of technological control which served to almost entirely remove any freedom of action and risk of violation of activity rules by participants.

Technological controls included a purpose-built bungy tower, fixed static lines that do not require staff to make calculations for participants' weight and falling distance, the use of anklets where both legs are independently tied together, and computer-controlled operation that allows manual 'let down' of client in the event of a power failure. Behavioral control involved control of both staff and client behavior, notably the fact that staff do not need to calculate weights and other measures (as is required for 'tailored' bungy jumping operations), and the use of carefully adhered to procedures for every aspect of the operation and every eventuality. Staff must also undertake computer-generated training that leads to 'Jumpmaster' qualifications. The 
respondent argued that this operation had no uncontrolled risks, and described the operation as a 'McDonalds' bungy jump, as it is a high volume, low-risk version of the tailored bungy jumps offered in Northern Queensland and New Zealand.

A very similar high-control approach to client safety was outlined by the bridge climbing respondent. For this activity, both technological and behavioural control are managed through the use of safety systems, notably the use of belt-clips that harness all clients to a rail throughout all stages of the bridge climb. Clients also received training through a mini climb over a purpose-built elevation within the operation's premises, prior to commencing the bridge climb proper. Other behavioral controls included the compulsory wearing of overalls and removal of any object from the bodies of participants, including handkerchiefs, to eliminate the risk of objects dropping onto the road below the bridge.

Control factors shared by both bungy jumping and bridge climbing operations included the opportunity for clients to pull-out of participation at any stage (prior to actually jumping for bungy jumping, of course), and the use of language cards, pictorials and signage to manage language and communication problems with overseas and non-English speaking clients. Both bungy jumping and bridge climbing respondents noted that the perception of risk is the important variable in their success as an adventure operator, and that many of the behavioral and technological controls ironically serve to heighten the level of perceived risk, while reducing actual risk to near-zero.

A contrasting approach to the control of technological and human risk was described by the operator offering flatwater kayaking and rollerblading activities. Indeed, it is apparent that for these activities, there are only limited technological and behavioral controls available to the 
operator, and consequently considerable personal freedom for the participant. In the case of rollerblading, for example, the operator noted that this activity was contracted out to a highly experienced instructor who insisted on use of all appropriate safety gear and techniques among participants. This activity was described as the most risky of a suite of activities offered by the operator, although it carried the lowest level of perceived risk, perhaps explaining the tendency of some participants to violate safety instructions once out of sight of the instructor. Hence, while appropriate technological (safety gear), behavioural (technique/following instructions), and locational (sticking with the correct route/staying with the group) controls were applied by the operator, the risk of client injury was still significant due to the limitations of these controls and the relative ease with which participants could negate the intended effects of these controls.

\subsection{A Model for Adventure and Ecotourism Dimensions of Control}

Figure 1 shows these technological and behavioral control factors, together with a third dimension of client injury control: locational control - or control over the place where the activity takes place. This dimension of control is included as ability to control the environment the activity takes place in will impact on the presence and awareness of hazards, and the ability to eliminate, isolate or minimize hazards, through housekeeping, environmental design, supervision, and other measures designed to control the activity environment.

\section{Figure 1 about here}

Clearly, these three dimensions of control interact significantly in creating the conditions for client safety during participation in adventure activities. The different activities provided by operators who were interviewed are positioned at the relevant point of interaction for the three dimensions. Hence, bungy jumping, with its high level of technological, behavioral and 
locational control is positioned at the intersection of the three high aspects of control, while rollerblading, with its relatively low level of control for each dimension, is positioned at the intersection between the low and moderate aspects of technological, behavioural, and locational control. Whale watching is considered moderate for all three dimensions of control, as operators can control client behavior (e.g. moving around the boat in choppy conditions) only to a certain degree, and close supervision may be required at certain points of the boat (e.g. on the front viewing deck) to avoid injuries due to loss of balance or clients falling overboard. Technological control is again moderate, and relates largely to aspects of boat design. Locational control is also moderate, as participants' location during the activity is only somewhat under the influence of the operator, and participants move through a variety of locations during the course of the activity (e.g. on the quayside, getting on and off the vessel, moving around the boat). Kayaking positioning in terms of control is largely dependent on whether it is unguided kayaking (kayak hire with little or no supervision) or guided kayaking. For unguided kayaking, the only measure of control is technological (kayak design and related gear), while guided kayaking (not shown in Figure 1) has control over location and to some extent over behavior, as participants are supervised as part of a group and may received some training, and would therefore occupy a similar position to guided rock climbing on the graphic shown in Figure 1. Instructed or guided rock climbing is positioned at a moderate level for all 3 dimensions, although operators may have a relatively high control level on each dimension in some situations.

A further dimension of control, safety culture and work organisation, is also included in Figure 1, and should always form part of the general analysis of risk and control for each adventure activity. Indeed, different elements of this dimension were discussed by interview respondents, as well as being identified as risk factors in the operator survey. Hence, factors such as having 
appropriate client/guide ratios, effective leadership, good communications, sensible operating decisions, an effective reporting culture, and a reasonable balance between safety and productivity all have a major influence on client safety, even where other aspects of control are high and well-managed. For this reason, this aspect of control may be most crucial in the management of client safety, and applies to all adventure and ecotourism activities, and in particular those activities where other aspects of control may be difficult to manage.

\section{Discussion and Conclusions}

This study has provided an initial exploratory analysis of the injury experience and safety management practice for Queensland adventure and ecotourism operations, providing further understanding to what is known from the extant literature on overseas visitor morbidity and mortality in relation to Queensland adventure and ecotourism. The study has produced new insights into the nature of the client safety problem from the operator perspective, for a broad cross-section of the sector, and some detail on practices currently used to manage client safety. Furthermore, the study has built on previous New Zealand-based surveys published in this journal, allowing a degree of comparison to be made between Queensland and New Zealand samples, as well as with epidemiological findings from the Australian literature. The study has also provided important insights into the usefulness of the online approach to data collection employed in this study. Indeed, comments from non-respondents suggested a shorter questionnaire, improved information about the survey and its purpose, and adjustments to the timing of the survey may serve to significantly improve participation levels among Queensland operators for future surveys, as part of an ongoing adventure and ecotourism monitoring and surveillance system. 
A notable finding from the present study was the low level of reporting of serious client injuries among the majority of adventure and ecotourism operators, with less than one serious harm injury per operator. Indeed, findings from the Australian epidemiological literature (Parfitt, 2006; Wilks and Coory, 2002) indicate that there may be serious under-reporting of client injury in this study, suggesting a poor reporting culture in some businesses. In most respects, however, reporting rates were similar to those found for New Zealand surveys, with similar numbers of injuries reported per operator, and almost identical ratios of minor to serious harm injuries. The validity of operators' reports of client injuries was supported to some extent by the fact that a healthy proportion of minor injuries (approximately eight) were reported for every serious injury. Perhaps the most important implication from this study is that operators should seek to improve their minor and near-miss reporting if they are serious about learning from their incident data (Bentley \& Page, 2008; Bentley et al., 2007), as these low-level incidents often have identical contributory causes as those which, often by chance alone, turn into serious or even fatal injuries.

Factors identified from the survey which may contribute to the relatively low reported injury rates include the low use of seasonal employees and low staff turnover relative to the New Zealand operator population, suggesting better skill and knowledge retention and development, and a relatively low proportion of overseas visitors compared with New Zealand, meaning higher levels of environmental familiarity and other supportive cultural factors such as language and communication issues among the Queensland clientele.

Turning to the issue of operators' perceptions of risk factors for injuries involving their clients, it is apparent from findings of this study, together with those undertaken in New Zealand, that injuries occur, not as a consequence of any single cause, but through an interaction of certain key factors. Foremost among these are adverse or changeable weather conditions, the presence of 
hazardous underfoot conditions, client unfamiliarity with the environment, and clients not following instructions. It is clear that these factors should feature strongly in operators' thinking when undertaken risk assessments for their activities, and should also be the focus of preventive efforts. Specifically, operators should seek to better manage the risk of slips, trips and falls among their activity participants, through better route planning (where applicable), ensuring the use of appropriate footwear, and consideration of speed of movement in adverse conditions. It is noted that a number of conceptual models have been produced based on the findings of New Zealand studies (e.g. Bentley \& Page, 2008; Bentley, Page, Meyer, Chalmers, \& Laird, 2001) that apply equally well to the Queensland context, and seek to illustrate the interaction between a wide range of contributory factors in the client injury process. These models can be useful in developing risk management programs that address all aspects of client risk, and can help operators identify the types of hazard areas they should seek to identify, assess and control, as appropriate for their activity.

This study has indicated that operators do not universally practice a number of important safety management activities, notably incident and injury investigation, where Queensland operators less commonly reported undertaking investigations for all client injuries than New Zealand operators. A more positive finding was that most operators $(80 \%)$ felt they would have all necessary systems in place if audited today, although this was somewhat offset by the fact that over half of the sample identified one or more crucial safety activity that needed to be implemented for their operation in the short to medium-term. Clearly it is desirable that adventure and ecotourism operators consistently apply all areas of safety management, and this remains an important focus for future research with the wider Queensland operator population. 
The survey and interview findings suggested a number of further broad implications for the management of client injury. For most operations, the level of behavioral, technological and locational control that can be utilized may be limited by the nature of the activity and other constraints. However, the management of client safety clearly depends on managing these aspects as effectively as possible, and this should be an important consideration as operators seek to balance the competing demands of offering a safe, yet exciting experience (Bentley \& Page, 2008; Bentley et al., 2001; Morgan \& Fluker, 2006). For many operations it is likely that work organization measures provide the greatest additional opportunity for improving risk management. Indeed, findings from this survey and those conducted in New Zealand have shown how aspects of work organization can play a role in client safety, notably issues such as guide/client ratios, hiring staff with appropriate levels of experience, sensible operating decisions, and good communication systems that are inclusive of non-English speaking clients, and allow effective exchange of information between guides and other staff, as well as with participants. The analysis of any given activity's risk should therefore be cognizant of organizational risk factors, along with those other aspects of injury control (technological, behavioural, locational) considered in this study. Moreover, operators should seek to develop a positive safety culture among staff (Reason, 1997) where management are committed to client safety; the balance between safety and productivity is appropriate; accidents and incidents are routinely reported and investigated fully; everyone knows what represents appropriate and inappropriate behavior in terms of client safety; and there are high levels of staff involvement in hazard and risk management activities, notably hazard identification and control.

An important limitation of the research was the relatively small numbers of participants in the on-line survey. However, this study was exploratory in nature, and was successful in terms of the 
wide array of adventure and ecotourism activities and regions represented in the sample. It was also an important aim of this study to establish the effectiveness of this method for collecting data on adventure and ecotourism injury data and safety management practice, towards the eventual development of an industry-wide client safety surveillance system for the State of Queensland and in the longer-term, an Australia-wide monitoring system. Furthermore, the analysis allowed for comparison with the New Zealand sample for many variables, with good agreement between findings providing a level of validation for the Queensland data. In addition, the use of a small number of face-to-face interviews allowed consideration of the perceptions of a number of non-respondents, and provided some detailed analysis of the differing safety and risk management practices of operators of very different forms of activity. Clearly, much more research is required if a reliable picture of the Queensland client safety experience and quality of safety management practice is to be achieved. This study has provided a starting point for this work, and the authors intend to build on this research in the development of an ongoing annual Queensland adventure and ecotourism safety monitoring exercise. 


\section{References}

Bentley, T. A., Meyer, D. Page, S. J., \& Chalmers, D. (2001). Recreational tourism injuries among visitors to New Zealand: an exploratory analysis using hospital discharge data. Tourism Management, 22, 373-381.

Bentley, T.A, Macky, K.,\& Edwards, J. (2006). Injuries to New Zealanders participating in adventure tourism and adventure sports: an analysis of Accident Compensation Corporation claims. New Zealand Medical Journal, 119, 1247.

Bentley, T.A., \& Page, S.J. (2001). Scoping the extent of adventure tourism accidents. Annals of Tourism Research, 28, 705-726.

Bentley, T.A., Page, S.J. (2008). A decade of injury monitoring in the New Zealand adventure tourism industry: A summary risk analysis. Tourism Management, 29, 857-869, Bentley, T.A., Page, S.J., Edwards, J. Monitoring injury in the New Zealand adventure tourism sector: An operator survey. Journal of Travel Medicine, in press.

Bentley, T., Page, S.,\& Laird, I. (2001). Accidents in the New Zealand adventure tourism industry. Safety Science, 38, 31-48.

Bentley, T. A., Page, S. J.,\& Laird, I. (2001). Safety in New Zealand's adventure tourism industry: The client accident experience of adventure tourism operators. Journal of Travel Medicine, 7, 239-245.

Bentley, T.A., Page, S.J.,\& Laird., I. (2003). Managing tourism safety: The experience of the adventure tourism industry. In: Wilks, J. and Page, S.J. (Eds.). Managing tourist health and safety in the new millennium (pp. 85-100). Oxford: Pergamon.

Bentley, T.A., Page, S.J.,\& Macky, K. (2007). Adventure tourism and adventure sports injury: The New Zealand Experience. Applied Ergonomics, 38, 791-796. 
Bentley, T., Page, S.J., Meyer, D., Chalmers, D.,\& Laird, I. (2001). How safe is adventure tourism in New Zealand? An exploratory analysis. Applied Ergonomics, 32, 327-338.

Bentley, T.A., Page, S.J.,\& Walker, L. (2004). The safety experience of New Zealand adventure tourism operators. Journal of Travel Medicine, 11, 1-7.

Blackwell, A. and Page, S.J. (2003) Biting Midges and Tourism in Scotland. In: Wilks, J., Page, S.J. (Eds.). Managing Tourist Health and Safety in the New Millennium (pp. 67-84). Oxford: Pergamon.

Bradley, C., \& Harrison, J. (2008). Hospital separations due to injury and poisoning, Australia 2004-05. Injury Research and Statistics Series Number 47 (Ca. no. INJCAT 117). Adelaide: AIHW.

Buckley, R. (2000) Neat trends: Current issues in nature, Eco - and adventure tourism International Journal of Tourism Research, 2, 437-444

Cater, C. I. (2006). Playing with risk? Participants' perceptions of risk and management implications in adventure tourism. Tourism Management, 27, 317-325.

Coxon, C. Safety in the dive tourism industry in Australia. In: J. Wilks, D. Prendergast (check the spelling - different to the text), P. Leggat (Eds.). Tourism in Turbulent Times (277-289). Oxford: Elsevier.

Deutskens, E., Ruyter, K. D. Wetzels, M.and Oosterveld, P. (2004) Response rate and response quality of Internet-based surveys: an experimental study. Marketing Letters, 15:1: 21-37 Ewert, A., and Jamieson, L. (2003). Current status and future directions in the adventure tourism industry. In: Wilks, J., Page, S.J. (Eds.). Managing Tourist Health and Safety in the New Millennium (pp. 67-84). Oxford: Pergamon.

Fennell D (1999). Ecotourism. Routledge, London 
Garrod B and Gössling S (eds.) (2007) Diving Tourism: Experiences, Sustainability, Management. Oxford: Elsevier .

Hall, C.M., and McArthur, S. (1994). Commercial white water rafting in Australia. Australian Journal of Leisure and Recreation, 1, 25-30.

Kittleson, M. (1997). Determining effective follow-up of e-mail surveys. American Journal of Health Behavior, 21(3), 193-196.

Morgan, D. (2006). Surf beach risk and safety. In: J. Wilks, D. Prendergast, P. Leggat (Eds.). Tourism in Turbulent Times (PP. 217-229). Oxford: Elsevier.

Morgan, D.,\& Fluker, M. (2006). Risk management for Australian commercial adventure tourism operation. In: Mansfeld,Y., Pizam A. (Eds.). Tourism, Security and Safety: From Theory to Practice (pp. 153-168).Oxford: Elsevier.

Mühlhäusler, P and Peace, A. (2001) Discourses of ecotourism: the case of Fraser Island, Queensland Language \& Communication, 21, 359-380.

Nichol, J. Wilks, J., \& Wood, M. (1996). Tourists as inpatients in Queensland Regional hospitals. Australian Health Review, 19, 55-72.

Page, S.J., Bentley, T.A.,\& Walker, L.(2005). Tourist safety in New Zealand and Scotland. Annals of Tourism Research, 32, 150-166.

Page, S. J., Bentley, T.A.\& Walker, L. (2003). Scoping the nature and extent of adventure tourism operations in Scotland: How safe are they?' Tourism Management 26 (3): 381-397 Page, S.J. (1997) The cost of adventure tourism accidents for the New Zealand tourism industry Tourism Policy Group, Ministry of Commerce, Wellington 
Page, S.J., \& Meyer, D. (1996). Tourism accidents: An exploratory analysis. Annals of Tourism Research, 23, 666-690.

Pearce, P. (2004) Assessing market convergence and divergence: studies of visitors to Australia's Great Barrier Reef. Paper presented at Tourism: State of the Art II conference, Glasgow, June 2004

Peattie, S., Clarke, P., Peattie, K. (2005). Risk and responsibility in tourism: Promoting sunsafety. Tourism Management, 26, 399-408.

Ryan, C. (1998) Saltwater Crocodiles as Tourist Attractions. Journal of sustainable tourism, 6, p. 314

Scott, D., Hockey, R., Spinks, D., Pitt, R. (2004). Water-related injury in Queensland. Queensland Injury Surveillance Unit, No. 85, December 2004.

Ryan, C. (2003). Risk acceptance in adventure tourism - paradox and content. In: Wilks, J., Page, S.J. (Eds.). Managing Tourist Health and Safety in the New Millennium (pp. 55-66). Oxford: Pergamon.

Statistics New Zealand (2007) Monthly overseas visitor arrivals by country of residence. Statistics New Zealand, Wellington.

Trouteaud, A.R. (2004) How You Ask Counts: A Test of Internet-Related Components of Response Rates to a Web-Based Survey. Social Science Computer Review; 22; 385

Tourism Queensland (2003) The Queensland Ecotourism Plan 2003-2008. Tourism Queensland, Brisbane, Australia. 
Tourism Queensland (2007a) Adventure Market Snapshot. Tourism Queensland, Brisbane, Australia.

Tourism Queensland (2007b) Queensland Regional Update - Year Ended December 2006

Tourism Queensland, Brisbane.

VisitScotland (2007) Tourism in Scotland 2007. Visit Scotland: Edinburgh.

Weaver, D. (2002). Hard-core Ecotourists in Lamington National Park, Australia. Journal of Ecotourism, 1, 19-

Wilks, J. (2000). Scuba Diving and Snorkeling Safety on Australia's Great Barrier Reef. Journal of Travel Medicine, 7, 283-299.

Wilks, J.\& Atherton, T. (1994). Health and safety in marine tourism: A social, medical and legal appraisal. Journal of Tourism Studies, 5, 2-16.

Wilks, J. \& Coory, M. (2000). Overseas visitors admitted to Queensland hospitals for waterrelated injuries. Medical Journal of Australia, 173, 244-246.

Wilks, J. \& Coory, M. (2002). Overseas visitor injuries in Queensland hospitals: 1996-2000. Journal of Tourism Studies, 13, 2-8.

Wilks, J., de Nardi, M., Wodarski, R. (2007). Close is not close enough: Drowning and rescues outside flagged beach patrol areas in Australia. Tourism in Marine Environments, 4, 57-62.

Wilks, J.\& Davis, R. (2000). Risk management for scuba diving operators on Australia's Great Barrier Reef. Tourism Management, 21, 591-599.

Wilks, J., Pendergast, D., Wood, M.T. (2002). Overseas visitor deaths in Australia: 1997-2000. Current Issues in Tourism, 5, 550-557. 
Yu, X. \& Weiler, B. (2001) Mainland Chinese Pleasure Travelers To Australia: A Leisure Behavior Analysis. Tourism, Culture \& Communication, 3, 81-?? 


\section{Adventure and ecotourism safety in Queensland: Operator experiences and}

\section{practice}

\section{Captions}

Table 1. Summary of most frequently identified risk factors, and comparison with the proportion of respondents identifying each factor in the most recent New Zealand survey of adventure tourism operators

\begin{tabular}{|c|c|c|}
\hline Risk factor & $\begin{array}{l}\text { Proportion of } \\
\text { respondents } \\
\text { selecting factor } \\
(\%)\end{array}$ & $\begin{array}{l}\text { NZ study } \\
\text { comparison } \\
(\%) \\
\end{array}$ \\
\hline \multicolumn{3}{|l|}{ Client-related } \\
\hline Clients not following instructions & 26 & 67 \\
\hline Clients not understanding instructions (language) & 10 & 18 \\
\hline Horseplay/showing-off & 16 & 20 \\
\hline Level of client skills and abilities & 12 & 46 \\
\hline Level of client fitness/health & 20 & 33 \\
\hline Client experience/familiarity with the task & 12 & 22 \\
\hline Client familiarity with the environment & 30 & 40 \\
\hline Client choice of clothing/footwear & 15 & - \\
\hline Client taking unnecessary risks/short-cuts & 12 & - \\
\hline Other & 12 & - \\
\hline \multicolumn{3}{|l|}{ Task and equipment-related } \\
\hline Activity difficulty level/degree of challenge & 10 & - \\
\hline Travelling to and from the activity/transit & 5 & - \\
\hline Unusual/unpredicted activity events & 40 & - \\
\hline Environment-related factors & & - \\
\hline Adverse or changeable weather conditions & 40 & 47 \\
\hline Hazardous underfoot terrain & 20 & 33 \\
\hline Hazardous overhead conditions & 5 & - \\
\hline Hazardous wilderness conditions & 5 & 28 \\
\hline Exposure to water/drowning threat & 15 & 21 \\
\hline Rips/water currents & 5 & - \\
\hline Exposure to sun & 32 & - \\
\hline Exposure to dangerous creatures & 15 & - \\
\hline Insect bites & 16 & - \\
\hline Other & 13 & \\
\hline \multicolumn{3}{|l|}{ Organisational/Management-related } \\
\hline Time pressure to complete activity on schedule & 15 & - \\
\hline Fatigue/stress due to long working periods & 7 & - \\
\hline Insufficient client/guide ratios & 2 & 3 \\
\hline Team dynamics & 7 & - \\
\hline Communication problems & 9 & 3 \\
\hline Ability to recruit experienced and qualified guides & 19 & 13 \\
\hline Pressure to operate in sub-optimal conditions & 8 & - \\
\hline Failure to identify, assess, control hazards & 12 & - \\
\hline Other & 12 & - \\
\hline
\end{tabular}


Table 2. Distribution of Injury event types for Queensland and New Zealand samples

\begin{tabular}{|l|l|l|}
\hline Injury event type & $\begin{array}{l}\text { Proportion of } \\
\text { respondents } \\
\text { reporting event } \\
\text { type } \\
\mathbf{( \% )}\end{array}$ & $\begin{array}{l}\text { NZ study } \\
\text { comparison } \\
(\%)\end{array}$ \\
\hline Slips, trips and falls on the level & 52 & 45 \\
\hline Falls from a height & 0 & 16 \\
\hline Manual handling & 5 & 1 \\
\hline Struck by an object & 5 & 7 \\
\hline Striking against an object & 10 & 27 \\
\hline Hand or limb caught in machine or object & 5 & 2 \\
\hline Vehicle or vessel is hit/crashes & 8 & - \\
\hline $\begin{array}{l}\text { Foot or ankle injury due to treading on something or } \\
\text { twisting ankle }\end{array}$ & 22 & 29 \\
\hline Drowning/non-fatal submersion & 5 & 6 \\
\hline Exposure to heat or cold & 5 & 13 \\
\hline Injured by animal & 13 & 9 \\
\hline
\end{tabular}

Table 3. Safety management activities used in the operations surveyed

\begin{tabular}{|l|l|}
\hline Safety/risk management activity & $\begin{array}{l}\text { Proportion of respondents undertaking this } \\
\text { activity (\%) }\end{array}$ \\
\hline Risk assessment of activities & 82 \\
\hline Safety audits or reviews & 60 \\
\hline Staff/guide training & 75 \\
\hline Written, audio or video information pre-activity for clients & 50 \\
\hline Regular maintenance checks on plant, vehicles, equipment & 82 \\
\hline Formal emergency plan/procedures & 82 \\
\hline
\end{tabular}


Figure 1. Dimensions of control for client safety for adventure and ecotourism operators

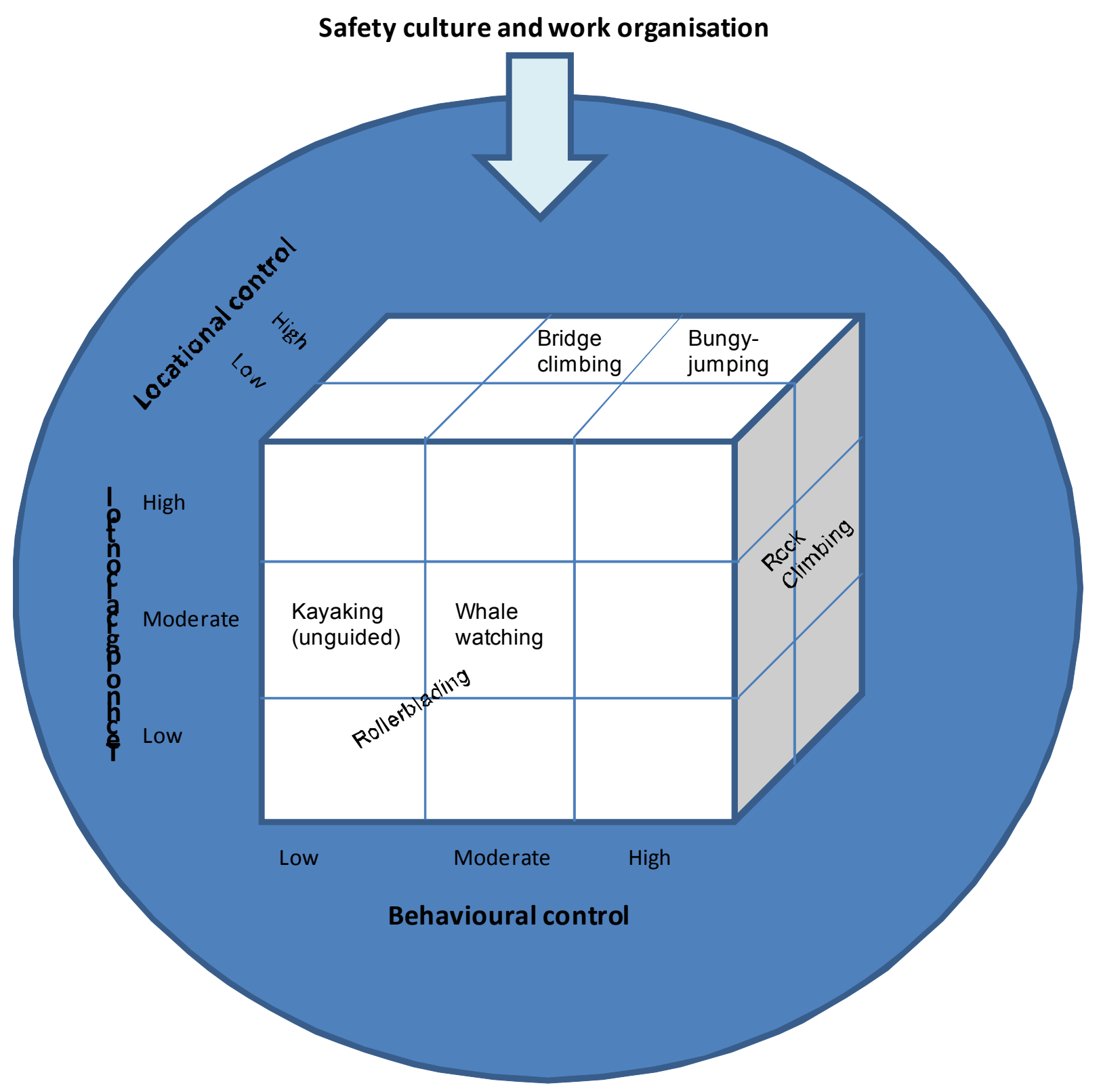




\section{Adventure and ecotourism safety in Queensland: Operator experiences and practice}

Tim A Bentley*, ${ }^{,}$, Carl Cater, ${ }^{2}$, Stephen J. Page, ${ }^{3}$

1) Department of Management and International Business

Massey University, Albany

New Zealand

Tel: 6494140800 extn: 9578

Fax: 6494418109

Email: T.A.Bentley@massey.ac.nz

2) Department of Tourism, Leisure, Sports and Hotel Management Griffith Business School, Griffith University

Gold Coast Campus, Queensland 4222

Australia

Email: C.Cater@griffith.edu.au

3) Department of Marketing

University of Stirling

Stirling

Scotland FK9 4LA

Email: S.j.page@stir.ac.uk

Corresponding author*

\section{Acknowledgements}

The authors would like to thank Queensland's adventure tourism operators and their employees who have given their time to participate in this survey. Thank you also to Tourism Queensland and the Queensland Outdoor Recreation Federation who providing considerable input to the study and assisted in gaining access to survey participants. The reviewers of this paper are also thanked as their helpful comments greatly strengthened the paper. 